\title{
Signal and Image Processing with Side Looking Sonar
}

\author{
R.Sathishkumar, \\ Department of Electronics and \\ Communication Engineering, \\ KL University, India
}

\author{
T.V.S.Prasad Gupta \\ Department of Electronics and \\ Communication Engineering, \\ KL University, India
}

\author{
M.Ajay Babu \\ Department of Electronics and \\ Communication Engineering, \\ KL University, India
}

\begin{abstract}
Mines placed on the sea floor are still a vast threat in civil and military shipping. This potential risk is typically encountered by advanced sonar signal processing techniques and time consuming manual evaluation of the sonar data by a human operator. Due to mission specific time constraints a computer aided or even autonomous analysis of the huge amount of data is desired. Acoustic waves suffer much less in seawater and therefore sonar sensors are the prevalent to be used in under water. A main difficulty is to extract information from side looking sonar (SLS). An overview of SLS approaches to underwater imaging is given in this paper. A short outlook to the system operation is also presented. This paper proposes a data model to generate SLS images that is suitable for identifying regions of interest $(\mathrm{ROI})$ in sonar images, in particular the detection of anomalies on the sea floor with focus on proud ground mines. It also presents a method to classify maps of the seafloor from sonar imagery. The techniques described are directly applicable to a range of remote sensing problems.
\end{abstract}

Keywords: Sonar, Image Processing, Data Model, Acoustic

\section{INTRODUCTION}

Side looking sonar (SLS) is towed from a survey vessel capture hundreds of meters of seafloor on each side. The near photographic quality images produced with its ability to map large areas of seafloor make it an essential piece of kit for high definition images of the seabed [1]. The purpose of a SLS survey is to determine the geographical location of targets or seabed features. Also it is used extensively for many commercial and military applications. Some examples include search and rescue operations, pipeline and cable route surveys, mine detection, fish finding, wreck hunting, recovery of drowned victims, marine archaeology and geological surveys. SLS reflections of isolated small objects give no indication of shape or attitude [2]. Very strong reflectors may give multiple echoes along a bearing line, and are identified by being equispaced in range. The plan view does not show how high an object is, unless an acoustic shadow is cast, in which case the length of the acoustic shadow is related to the height of the object. SLS images have been computer processed using software originally developed for planetary and terrestrial imaging missions [3]. These general purpose programs were used to perform geometric corrections for slant range and ships speed as well as shading corrections for illumination gradients. Although these corrections are important steps in preparing the images for interpretation, they are typically insufficient to register the images to a cartographic base. This type of correction is a prerequisite in order to correlate SLS images with other data (e.g. bathymetry, geophysical data, samples, etc). The potential value SLS images are frequently unrealized since the output from sonar scan line recorders cannot be conveniently registered to a cartographic map base. It possesses many distortions inherent in the image scanning technique. Also, unless track lines are perfectly straight, the resulting image printed will poorly represent the "footprint" of the scan lines on the seafloor. When available as a map projection, SLS images can be straightforwardly and more conveniently compared to other map based data (for example, topography, sediment type and grain size, bottom currents, etc). SLS image distortions also account for the irreconcilable mismatches encountered in joining adjacent image swaths to create sonar mosaics [4]. The Figre. 1 shows the sonar beam and footprint.

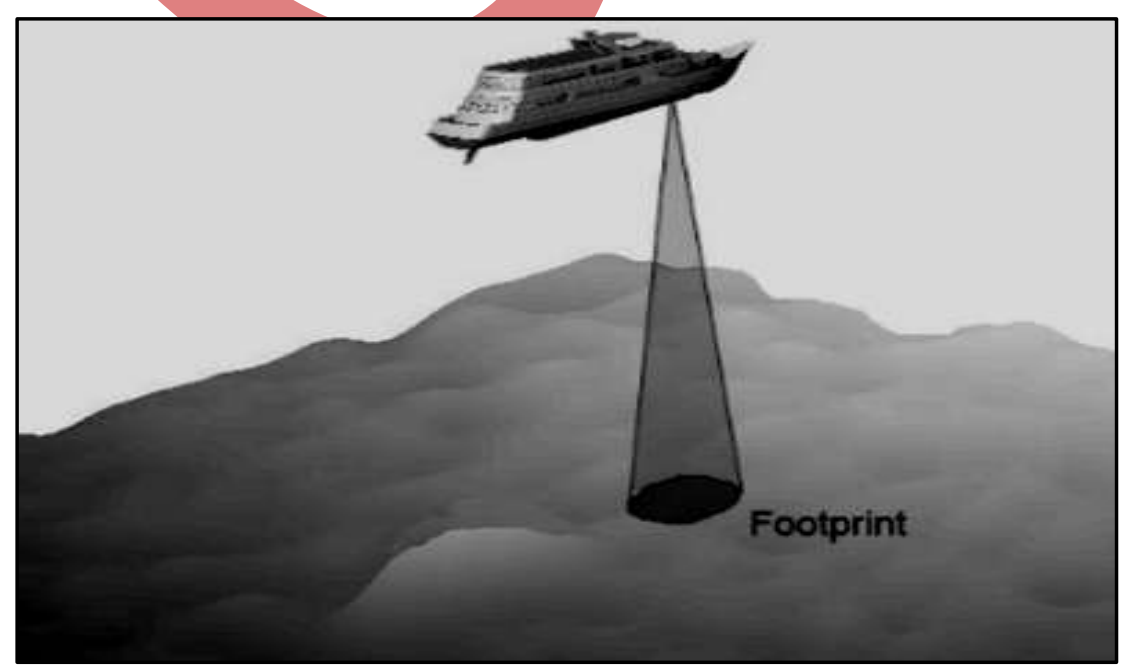

Figure.1 Sonar Beam and Footprint 


\section{SIDE LOOKING SONAR OPERATION}

There are three main type of SLS systems now used for internal investigations and commercial contracts: 1. Shallow water sonar system contains dual frequency SLS and bottom profiler. It has operation depth $100 \mathrm{~m}$ and uses multi wired tow cable. 2. Medium depth operated sonar system contains SLS, bottom profiler, fish mounted acoustic $(12 \mathrm{kHz})$ interrogator for ultra short base navigation system and compass system. It has operation depth $2000 \mathrm{~m}$ and uses single coax or shielded wire tow cable. 3. Deep tow sonar system is based on the mother ship and is equipped by SLS, bottom profiler and long base acoustic navigation transponder. It has operation depth $6000 \mathrm{~m}$ and uses single wire coax tow cable. The SLS is a powerful, versatile and low cost tool for surveying the sea floor. The sonar emits fan shaped signals perpendicular to the direction of travel. The signals scan a swath of sea floor from a point just below the towfish to a limited distance away from the line of travel on both sides [5]. The raw data are two time series of digitized sound: the backscattered signals from each ping on the port and starboard sides of the towfish. The sonar signal has a finite width, and it spreads as it travels. Therefore the footprint of one ping is actually a fan-shaped segment rather than a line. The area of the swath furthest from the towfish is actually scanned more than areas near the path of the towfish, because of the way the sonar beam spreads out. When the pixel is actually covered by more than one fan shaped footprint, it seems reasonable that we can introduce some weighting algorithm to combine the contributions of all pings into the pixel's intensity. However, it usually causes smoothing effect, which makes the patched lines themselves become false features. The main reason for this is the background intensities of images are generally with high frequency noises. transmits a narrow fan shaped acoustic pulse perpendicular to its direction of travel [6]. As the acoustic pulse travels outward from the system, the seabed and other objects reflect some of the sound energy back in the direction of the sonar. The travel time of the returned pulse is recorded together with its amplitude as a time series and sent to a topside console for interpretation and display [7]. The topside console stitches together data from successive pulses, creating a long continuous image of the seafloor as the SLS is towed from a survey vessel.
It show echoes of objects that reflect sound back to the transducer, such that hard shiny surfaces are sometimes only seen when they are at right angles and rough seabed textures can blot out smaller targets completely. Materials, such as metals, boulders, gravel or recently extruded volcanic rock, are very efficient at reflecting acoustic pulses (high backscatter). Finer sediments like clay and silt, on the other hand, do not reflect sound well (low backscatter). Strong reflectors create strong echoes, while weak reflectors create weaker echoes. Knowing these characteristics, the strength of acoustic returns we can examine the composition of the seafloor and any objects. The Figure. 2 shows overview of SLS beam and the numbers on the diagram are 1.Depth 2. Vertical beam angle 3.Range 4.Swath width 5.Tow depth 6. Port and starboard channel separation 7.Horizontal beam width. The acoustic signal is transmitted and received by a pair of transducers, which are deployed in the towfish. The beam patterns show narrow horizontal directivity (114 $\left.\mathrm{kHz}: 1^{\circ}, 410 \mathrm{kHz}: 0.3^{\circ}\right)$ and wide vertical directivity (114 $\left.\mathrm{kHz}: 50^{\circ}, 410 \mathrm{kHz}: 40^{\circ}\right)$. The beams intercept the seafloor in thin stripes. The backscatter values from the short acoustic signal are recorded as time series across the stripes, revealing bathymetric and textural differences of the seafloor. The image is built up with consecutive transmission pings along the travel path of the sonar. The best image is achieved at low grazing angles of incidence; a ratio of $1: 10$ between fly height above the seafloor and desired coverage is recommended. To get the towfish to the corresponding optimum depth a ratio of up to $1: 4$ between towfish depth and tow cable length is needed. Although mainly providing imaging, relative bathymetry information and object dimensions can be derived from the data. Post processing of the acquired data leads to improvement of imaging quality. Geo-referencing allows production of image maps, called side scan mosaics. Normalisation algorithm, making use of beam pattern and backscatter information, lead to automated texture classification, allowing the differentiation of bottom types and objects [8]. For optimal post processing and normalisation Sonar acquires data with 24 bit sample depth, which is equivalent to a dynamic range of $144 \mathrm{~dB}$. This includes the complete range of underwater acoustics signals from background noise to cavitation.

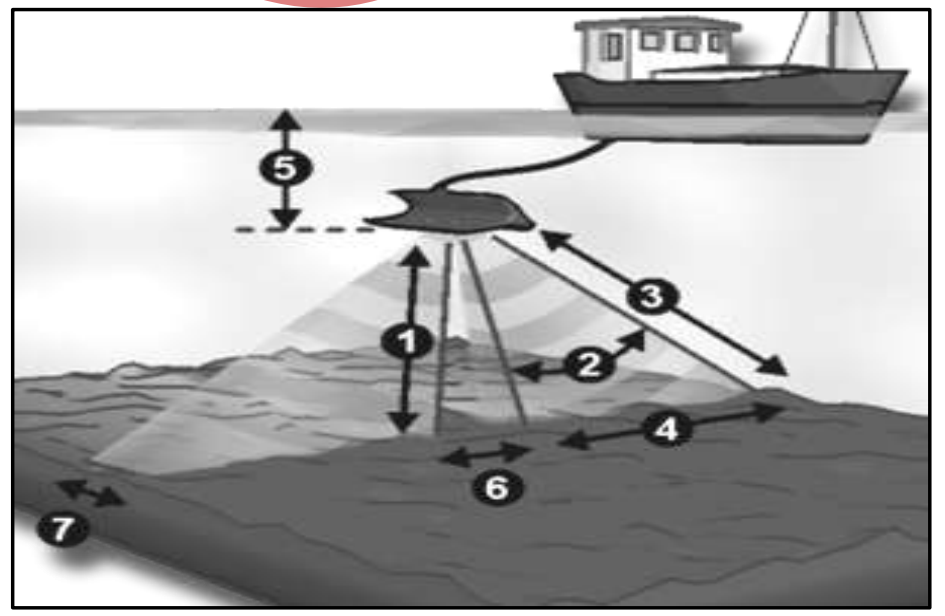


Figure.2 Overview of SLS Beam

The energy broadcast by the sonar array should be exactly the same for each ping. However, as the towfish is pulled under the water, it is constantly moving because of waves and currents. This causes the energy actually incident on the sea floor to vary from ping to ping. For example, rolling motion might cause the energy level to rise on one side and drop on the other side.

The level of backscattered energy also changes with the surface character of the seabed. To suppress this kind of noise, each ping should have a similar energy level to the pings before and after it [9]. For each ping, we find the average energy level of the 20 pings before and after it. This average is used as a reference value, and the total energy of the ping is smoothed to match it [10]. The ping energy level does vary in reality when the character of the sea floor changes. That is why the smoothing must be done with reference to a fairly short sequence of pings. If the smoothing is too extensive, real changes in the sea floor will be obscured. Moreover the number of pings is not an independent variable proportional to the real space occupation [11]. The surveying speed and footprint's width are also involved, which makes the criteria for reference window definition quite complicate [12].

\section{IMAGE FORMATION MODEL}

The geometry of the image formation process for a SLS is shown in Figure.3. The sonar's acoustic source at $O$ produces an ensonification pulse that illuminates the seafloor. Some of the acoustic energy reaching any seabed point $p$ is scattered back and can be measured by the sensor. The intensity of the corresponding pixel on the image will be proportional to the amount of energy scattered back from the surface point. The illuminating pulse is not isotropic, but follows a particular beam profile $\Phi$ that depends on the grazing angle a subtended by the vector $r$ from $O$ to surface point $p$. In order to model the scattering process the traditional Lambertian model permits to derive the returned intensity from the parameters defining the observed scene. This simple model for diffuse scattering assumes that the returned intensity depends only on the local angle of incidence $\theta$ of the illuminating sound pulse, and not on the direction of observation or on the frequency of the pulse. For the problem to be manageable the surface describing the observed scene has to be univalued. The intensity

$$
I(r)=K \Phi(r) R(r) \frac{n . r}{\|n\|\|r\|}=K \Phi(r) R(r) \cos (\theta(r))
$$

Where $\Phi$ represents the intensity of the illuminating sound wave at point $p, R$ is the reflectivity of the seafloor, $\theta$ is the incidence angle of the wave front and $\mathrm{K}$ is normalization constant. Since most logged side-looking images already include some kind of intensity correction, all the intensity variations caused by the sensor's beam profile, the spherical spreading loss and the TVG and other corrections are supposed to be grouped under the beam pattern $\Phi$. The coordinate system centered at the sensor in $O$, the $x$ axis being the across track ground distance and y pointing along the sensor's trajectory,

$r=(x, 0, Z(x, y))$

$n=\left(-\frac{a Z}{a x}(x, y),-\frac{a Z}{a y}(x, y), 1\right)$

Where the $y$ coordinate in $r$ is 0 because the SLS pulse $\Phi$ is shaped so that only the points contained in the $x-z$ plane are illuminated. Note that although this does not directly apply to the sonar pulses used for SA imaging, the resulting $S A$ images are to all practical purposes equivalent to a side-scan image with constant resolution in the range direction.

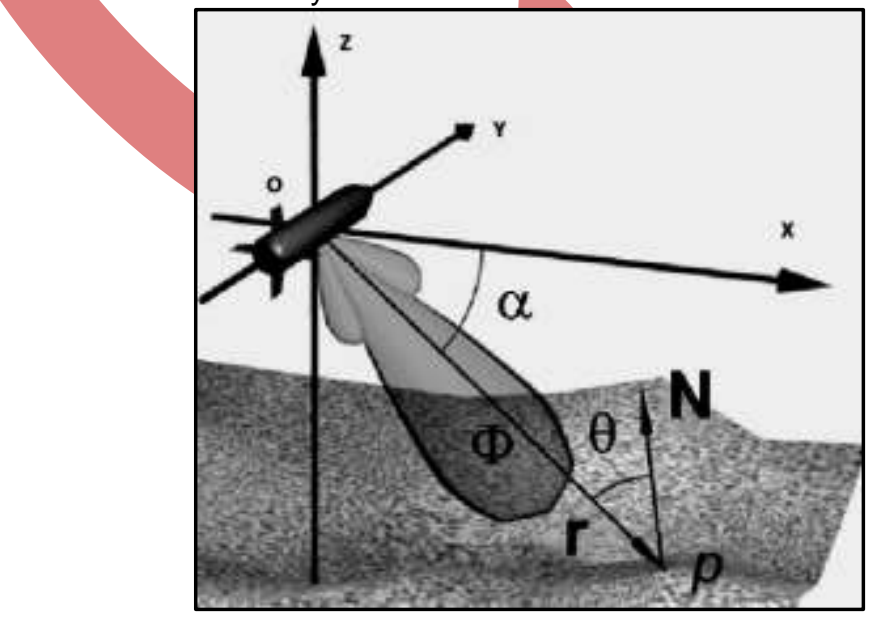

Figure.3 Data Model of SLS

Combination of expressions (1) and (2) yields the forward model for the computation of the intensity $I$ at any point $P$, given the model parameters $R, Z$ and $\Phi$ in ground range coordinates $x, y$ from the sensor.

$$
I(x, y)=K \Phi(x, y) R(x, y) \text {. }
$$


$\frac{Z(x, y)-x \cdot \frac{a Z}{a x}(x, y)}{\sqrt{x^{2}+Z^{2}(x, y)} \cdot \sqrt{\left(\frac{a Z}{a x}(x, y)\right)^{2}+\left(\frac{a Z}{a y}(x, y)\right)^{2}+1}}$

Where the surface gradients can be approximated by finite differences for and where the normalization value $\mathrm{K}$ is

$$
K(x, y)=\frac{Z \sqrt{x^{2}+Z^{2}} \cdot \sqrt{1+\left(\frac{a Z}{a y}\right)^{2}+\frac{x^{2}}{Z^{2}}\left(1+\left(\frac{a Z}{a y}\right)^{2}\right)^{2}}}{-Z^{2}-x^{2} \cdot\left(1+\left(\frac{a Z}{a y}\right)^{2}\right)}
$$

Where the explicit dependencies on $(x, y)$ have been dropped for clarity.

\section{BEAMFORMING}

Images based directly on the raw time series data have a blind zone in the center and needs to be processed into an image which roughly corresponds to a plane area of the sea floor. The most basic form of processing for the data is slant range correction in which the seabed is assumed as a perfect flat plane. The Figure. 4 shows the SLS operation at sea. Then, given the altitude of the towfish, and the time at which the backscatter reaches the sonar array, we can calculate a position on the seabed. After processing the data, the blind zone in the center of image disappears and every datum is relocated to a position more representative of the actual seabed. In general, the 16 bit raw data allows more flexible contrast exaggeration processing than the 8 bit data set.

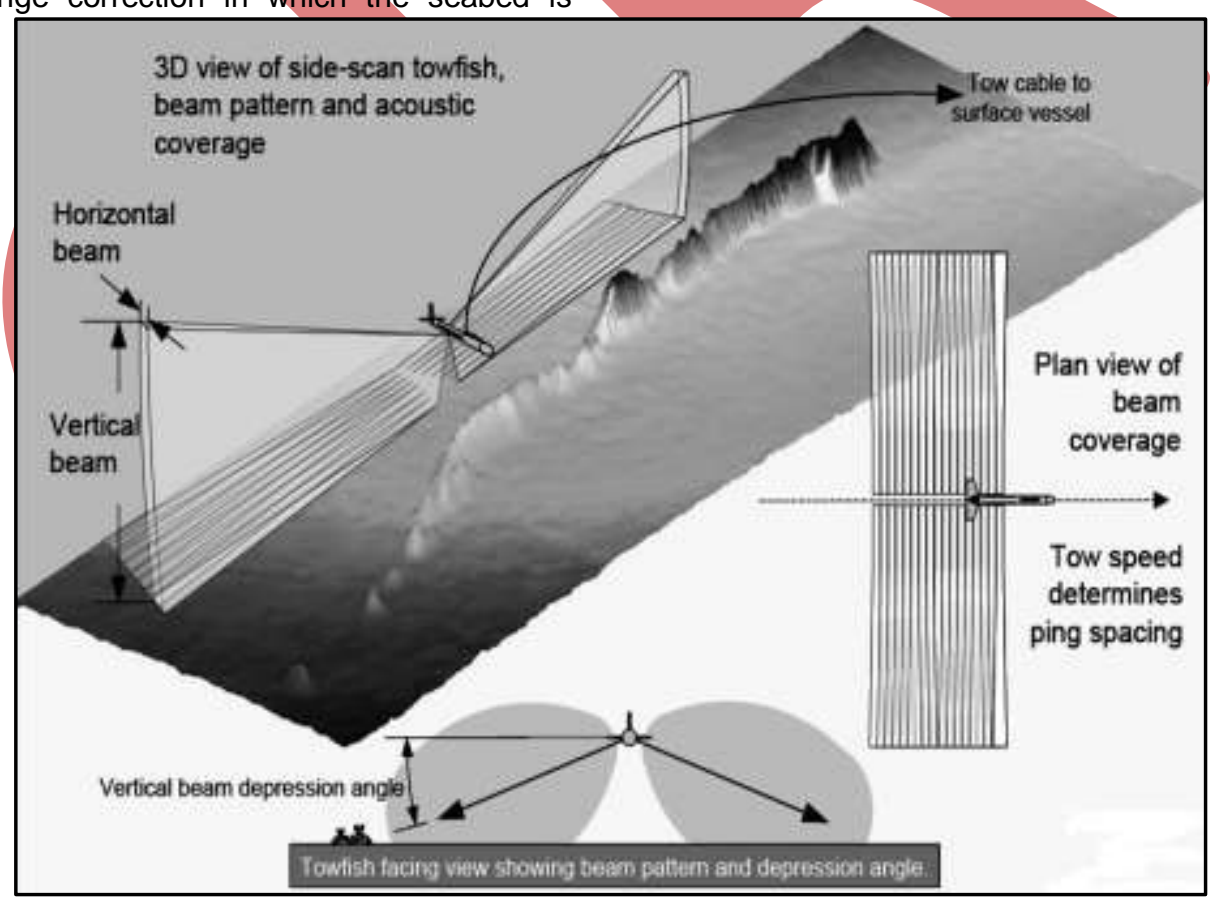

Figure.4 Overview of SLS operation at Sea

\section{RESULT}

The most prominent feature of SLS is the too strong intensity near the path of the towfish, and the weak response at the outer edge of the swath. Across the swath, the amount of energy hitting the seabed will vary with the distance from the towfish. The angle at which the wave hits the sea floor also varies. The raw data are the backscattered energy from the seabed; both the level of incident energy and grazing angle will affect the data. Since the SLS is two sided sonar, each side has its own main lobe pointed outward from the vertical line about 45 degrees, the maximum intensity therefore located around this angle, rather than right below the towfish. To compensate for the uneven illumination, we need to know the energy distribution function relative to the angle. One simple way to find the energy distribution is to sum up the energy levels for each angle over the whole data series. Using this statistical result, we can calculate the average energy for each angle; the inverse of this average can be applied as a correcting factor to individual data in the time series. Most processing procedures for the same image correction purpose are executed by time variable gains (TVG), either at source 
or in post processing. However, the time based functions are not suitable to describe the variation of backscattering energy which is basically controlled by the grazing angle.
The Figure.5 shows the SLS mapping that explains the acquired image, contrast adjustment, thresholding, classification and identification

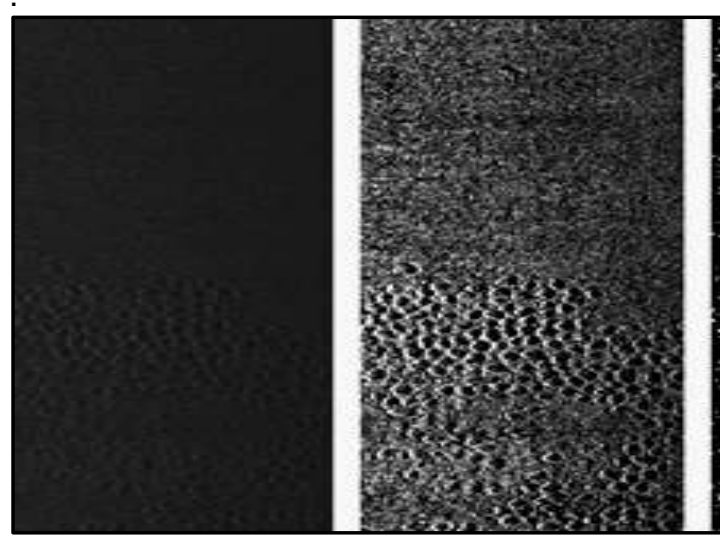

a) Acquision

b) Contrast Adjust

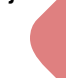

\section{CONCLUSION}

In this work, the relations between surface properties and the images resulting when the surface is observed by SLS (backscatter strength) are studied. The characterization of this sonar imaging process can be used in two ways: by applying the forward image formation model; conversely, by inverting the image formation model. The typical imaging model considered here is active SLS with a frequency of hundreds of kilohertz, which usually allows for sub-decimeter resolution in range and azimuth.

\section{ACKNOWLEDGEMENT}

We are grateful to the management of the KL University for providing the necessary facilities for the stimulating research environment. We received lot of support from Prof.Dr.Shree Hari Rao, Vice Chancellor of the KL University. We received a lot of guidance from Dr.Dhilsha Rajappan, Joint Project Director of National Institute of Ocean Technology-NIOT, India, who provided us the initial desire, interest and ideas on ocean engineering projects. We extend our thanks to Prof.Dr.Habibullakhan, Head of ECE department, KL University for his continual encouragement on the research work.

\section{REFERENCES}

1. Weiqing Zhu; Min Zhu; Xiaodong Liu; Feng Pan; Xiangjun Zhang; Yuling Wang; Changhong Wang; , "High resolution bathymetric sidescan sonar," OCEANS '02 MTS/IEEE, vol.1, no., pp. 223- 227 vol.1, 29-31 2002

2. E. Coiras, Y. Petillot, D.M. Lane, "MultiResolution 3D Reconstruction from Side- Scan Sonar Images", IEEE Transactions on Image Processing, Vol. 16, No. 2, pp 382-390, 2007

3. Pinto, M.; Ferreira, B.; Matos, A.; Cruz, N., "Side scan sonar image segmentation and feature extraction," IEEE OCEANS-2009, Global and Local Challenges, vol.1, no.1, pp 1-9, October 2009

4. Shuang Wei; Leung, H.; Myers.V, "An automated change detection approach for mine recognition using sidescan sonar data", IEEE International
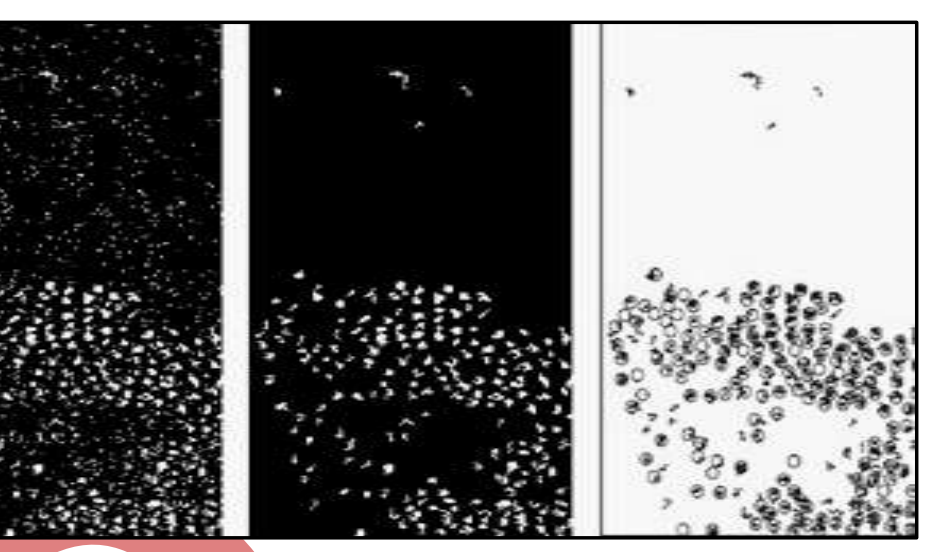

d) Classification

e) Identification

Conference on Systems, Man and Cybernetics, vol.1, no.1, pp553-558, 2009

5. R.Sathishkumar and A.Vimala Juliet, "Analysis of Marine Sensor Signals in Fast Time Domain Processing", International Journal of Wireless Communication, Vol. 1, No.5, pp 210 - 215 August 2009.

6. Jianhu Zhao; Weiliang Tao; Hongmei Zhang; Kun Yang; , "Study on side scan sonar image matching based on the integration of SURF and similarity calculation of typical areas," OCEANS 2010 IEEE - Sydney, vol.1, no.1, pp 1-4, May 2010

7. R. Sathishkumar, Prasad Gupta T.V.S, et al, "Challenges of visualizing the seafloor with modern sonar system" International Journal of Current Research, Vol.4, No.12, pp 529-532, December, 2012

8. Quintal, R.T.; Kiernan, J.E.; Shannon, J.; Dysart, P.S.; , "Automatic contact detection in side-scan sonar data", IEEE International Conference on Technologies for Homeland Security (HST), vol.1, no.1, pp 270-275, November 2010

9. Fawcett, J.; Myers, V.; Hopkin, D.; et al, "Multiaspect Classification of Sidescan Sonar Images: Four Different Approaches to Fusing Single Aspect Information," IEEE Journal of Oceanic Engineering, vol.35, no.4, pp.863-876, October 2010

10. Celebi, A.T.; Gullu, M.K.; Erturk, S.; , "Mine detection in side scan sonar images using Markov Random Fields with brightness compensation," IEEE 19th Conference on Signal Processing and Communications Applications, vol.1, no.1, pp 916-919, April 2011

11. Chen, E.; Sheng-Wei Huang; Wei-Han Wang; Jen Hwa Guo, "Side scan sonar grid map for Unmanned Underwater Vehicle navigation," OCEANS-2011, vol.1, no.1, pp 1-8, September 2011

12. Vandrish, P.; Vardy, A.; Walker, D.; Dobre, O.A.; , "Side-scan sonar image registration for AUV navigation," Underwater Technology (UT), 2011 IEEE Symposium on Scientific Use of Submarine Cables and Related Technologies, vol.1, no.1, pp 1-7, April 2011 